\title{
Comparision the Timing of Lower Limb Muscles Activity in the Stance Phase of Running Between Athletes with Reconstructed Anterior Cruciate Ligament and Healthy
}

\author{
Amin Mardazad ${ }^{1}$, Nader Farahpour ${ }^{2, *}$, Yasin Hoseini ${ }^{3}$ \\ ${ }^{1} \mathrm{MSc}$ in Sports Biomechanics, Faculty of Sport Sciences, Bu-Ali Sina University, Hamedan, Iran \\ 2 Professor, Faculty of Sport Sciences, Bu-Ali Sina University, Hamedan, Iran \\ ${ }^{3}$ Assistant Professor in Sports Biomechanics, Faculty of Literature and Humanities, Malayer University, Hamedan, Iran \\ * Corresponding author: Nader Farahpour, Professor, Faculty of Sport Sciences, Bu-Ali Sina University, Hamedan, Iran. E-mail: \\ naderfarahpour1@gmail.com \\ How to Cite this Article: \\ Mardazad A, Farahpour N, Hoseini Y. Comparison the Timing of Lower Limb Muscles Activity in the Stance Phase of Running \\ Between Athletes with Reconstructed Anterior Cruciate Ligament and Healthy. J North Khorasan Univ Med Sci. 2020;6(4):59-69. \\ DOI: 10.29252/ijrn-06047
}

\begin{tabular}{l}
\hline Received: 05 Nov 2019 \\
Accepted: 09 Mar 2020 \\
\hline Keywords: \\
Running \\
Anterior Cruciate Ligament \\
Electromyography \\
\hline C 2020 Iranian Journal of \\
Rehabilitation Research in Nursing
\end{tabular}

Rehabilitation Research in Nursing

\begin{abstract}
Introduction: After reconstructing the anterior cruciate ligament, to prevent re-injury of the ligament, evaluation of neuromuscular function is of clinical importance in coordinating the onset time and time to peak of lower limb's muscles activity. Aim: Therefore, the purpose of this study was to compare the timing of muscle activity in the stance phase of running between athletes with reconstructed anterior cruciate ligament and healthy subjects.

Methods: This study was a semi-experimental and applied type. Ten athletes with reconstructed anterior cruciate ligament who at least one year had passed since their surgery, as experimental group and ten healthy subjects as the control group of Hamedan city were volunteered to participate in the study. The control group, in terms of age, height, and weight, were homological with the experimental group. The surface electromyography system was used to quantify the onset time and time to peak of the lower limb's muscle activity. Multivariate MANOVA, with a significance level of $\mathrm{P}<0.05$, was used for statistical analysis.

Results: In the experimental group, the onset of the activity in tibialis anterior, medial gastrocnemius, vastus medialis, vastus lateralis, gluteus medius ( $P=0.001)$, as well as the time to peak in tibialis anterior, vastus lateralis, vastus medialis, gluteus medius, semitendinosus $(P=0.001)$ and biceps femoris $(P=0.045)$, were delayed.

Conclusions: During the stance phase of running, the experimental group displayed a delay in the activation onset and a longer time to peak in the lower limb's muscles. It is not clear if these changes are due to a neuromuscular adaptation or proprioception related damage. A more comprehensive study is recommended to clarify this aspect. It is recommended to assess the possible link between these delays with the reoccurrence of anterior cruciate ligament rupture.
\end{abstract}

\section{Extended Abstract}

\section{OBJECTIVE}

A nterior cruciate ligament rupture is more common knee injury in athletes and, in addition to joint mechanical instability, due to disturbance of the knee proprioception system, it causes poor balance and loss of athletic performance [2]. After reconstructed anterior cruciate ligament, the amount of activity and lifestyle changes [6], and there is often the risk of osteoarthritis and meniscus tear in these people [7]. Regarding the mechanism of anterior cruciate ligament rupture, it is believed that excessive mechanical loading at the knee simultaneous with rapid deceleration, abrupt change of direction during landing 
or running, are important risk factors for this injury [8, 9]. In maneuvers accompanied by changes in acceleration and direction of movement, the lower extremities may be in a locked position. In these conditions, excessive ankle pronation, internal tibial torsion, Knee valgus and external rotation of the thigh occurs that the anterior cruciate ligament is overloaded suddenly and these are the leading causes of ligament rupture [10]. Muscles are responsible for the distribution and absorption of these dynamic loads on the lower limbs [15]. At this stage, the nervous-muscular system decreases the mechanical forces applied to the knee by controlling the duration of the activity, the intensity of exercise, and the onset of use [16]. In the running, the lower extremity is continuously subjected to mechanical load stress, and this loading becomes more severe with fatigue and loss of muscle function. During running slowly, the loads fall below the physiological threshold level. However, sometimes due to mechanical conditions and inadequate muscle response when performing the maneuvers, the total exceeds overtime interval, and the rupture of the anterior cruciate ligament occurs [12]. When the anterior cruciate ligament is damaged or replaced by a graft, many of the primary mechanoreceptors and neural connections are not restored. In addition to the mechanical function of the anterior cruciate ligament, this ligament provides essential sensory information for understanding the joint status and detecting threshold motion and muscle reflex stability to maintain collective balance [44]. After reconstructing the anterior cruciate ligament, to prevent re-injury of the ligament, evaluation of neuromuscular function is of clinical importance in coordinating the onset time and time to peak of lower limb's muscles activity. Therefore the purpose of this study was to compare the timing of muscle activity in the stance phase of running between athletes with reconstructed anterior cruciate ligament and healthy subjects.

\section{MATERIALS AND METHODS}

This study was a semi-experimental and applied type. Ten athletes with reconstructed anterior cruciate ligament who at least one year had passed since their surgery, as experimental group and ten healthy subjects as the control group of Hamedan city were volunteered to participate in the study. The control group, in terms of age, height, and weight, were homological with the experimental group. Surface electromyography system was used to quantify the onset time and time to peak of lower limb's muscles (tibialis anterior muscle, medial gastrocnemius muscle, lateral gastrocnemius muscle, vastus medialis muscle, vastus lateralis muscle, semitendinosus muscle, biceps femoris muscle and gluteus medius muscle) activity in the stance phase of running. For both groups, each subject runs three times on an $18 \mathrm{~m}$ path in the lab at an average speed of $2.5 \mathrm{~m} /$ $\mathrm{s}(9 \mathrm{~km} / \mathrm{h})$. Running gear was calculated based on the kinematic data from the cameras. Each repetition of the tests was allowed if the individual moved at a specified speed and landed on the force plate device with the leg reconstructed. Multivariate MANOVA, with a significance level of $\mathrm{P}<0.05$, was used for statistical analysis.

\section{RESULTS}

In the experimental group, the onset of the activity in tibialis anterior muscle, medial gastrocnemius muscle, vastus medialis muscle, vastus lateralis muscle, gluteus medius muscle $(\mathrm{P}=0.001)$ as well as the time to peak in tibialis anterior muscle, vastus lateralis muscle, vastus medialis muscle, gluteus medius muscle, semitendinosus muscle $(\mathrm{P}=0.001)$ and biceps femoris muscle $(\mathrm{P}=0.045)$ were delayed.

\section{CONCLUSION}

During the stance phase of running, the experimental group displayed a delay on the activation onset and a longer time to peak in the lower limb's muscles. These delays may be related to the recurrence of anterior cruciate ligament or the occurrence of osteoarthritis. It seems that people with anterior cruciate ligament surgery due to proprioception abnormalities during injury as well as residual proprioception abnormalities after surgery and neuromuscular system changes have delayed onset of activity and time to peak muscle activity. Therefore, delays in the start of the event and the time to peak of the productive force of these muscles are risk factors. Periodically, Muscle rehabilitation should be taken seriously to increase muscle strength and coordination and Improvement proprioception receptors in these patients after surgery. It is not clear if these changes are due to a neuromuscular adaptation or proprioception related damage. A more comprehensive study is recommended to clarify this aspect. It is recommended to assess the possible link between this delay with the reoccurrence of anterior cruciate ligament rupture and occurrence osteoarthritis.

\section{Ethical Considerations}

This research was confirmed by the Ethics Committee of Medical Studies of the University of Medical Sciences Hamedan on 2/3/1394 with number 1199/9/35/16 / p. Subjects signed a consent form to participate in the study after being informed of the purpose and method of the research. Also, the information of the participants in this study was kept confidential.

\section{Funding or Supports}

There was also no funding or supports.

\section{Author's Contributions}

Mr. Amin Mardazad Navi: He did Writing and preparing the initial and final drafting, statistical analysis, submitting and paper revision, and laboratory work. Mr. Nader Farhpour: He did the initial idea of studying and checking the initial draft of the article. MR. YASIN HOSSEINI: He did the initial design of studying and paper revision and laboratory work.

\section{Conflict of Interest}


The authors of the article do not declare any conflict of interest.

\section{Applicable Remark}

This study will be useful for the rehabilitation of people after anterior cruciate ligament surgery and can also help people during running to prevent anterior cruciate ligament injury and osteoarthritis and ultimately a healthy lifestyle

\section{Acknowledgments}

This article is the result of Mr. Amin Mardazad's thesis with the supervisor of Dr. Nader Farahpour approved by the University of Bu Ali Sina Hamadan. This research was confirmed by the Ethics Committee of Medical Studies of the University of Medical Sciences Hamedan on $2 / 3 / 1394$ with number $1199 / 9 / 35 / 16 /$ p. We appreciate all the relevant Officials as well as all the athletes who participated in the study. Medical Studies of University of Medical Sciences Hamedan on $2 / 3 / 1394$ with number $1199 / 9 / 35 / 16 /$ p. We appreciate all the relevant Officials as well as all the athletes who participated in the study. 


\section{مقايسه زمانبندى فعاليت عضلات اندام تحتانى در مرحله اتكاى دويدن بين ورزشكاران با سابقه جراحى رباط متقاطع قدامى و سالم}

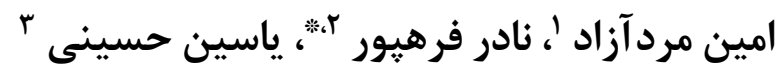

' كارشناسى ارشد بيومكانيك ورزشى، دانشكده علوم ورزشى، دانشكاه بوعلى سينا، همدان، ايران

r r استاد، تروه بيومكانيك ورزشى، دانشكده علوم ورزشى، دانشكاه بوعلى سينا، همدان، ايران ץ استاديار دانشكده ادبيات و علوم انسانى، كروه تربيت بدنى و علوم ورزشى، دانشخاه ملاير، همدان، ايران

" نويسنده مسئول: نادر فرهيور، استاديار، استاد، گروه بيومكانيك ورزشى، دانشكده علوم ورزشى، دانشكاه بوعلى سينا، همدان، ايران. ايميل: naderfarahpour1@gmail.com

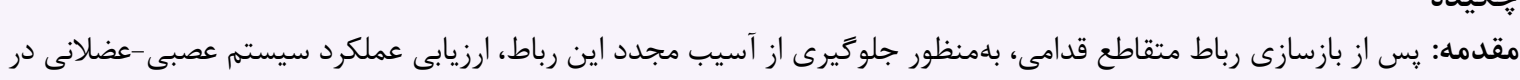

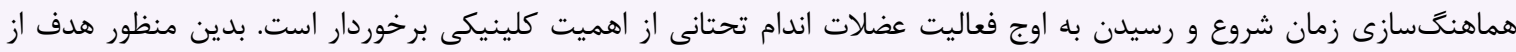

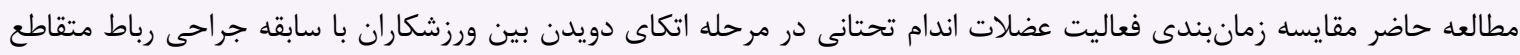

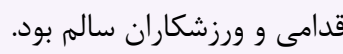

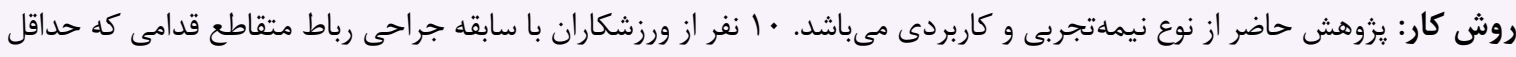

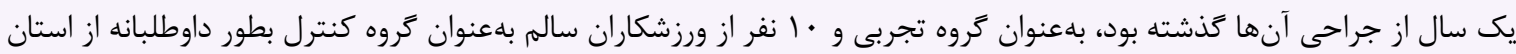

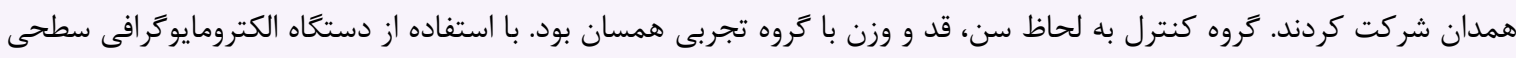

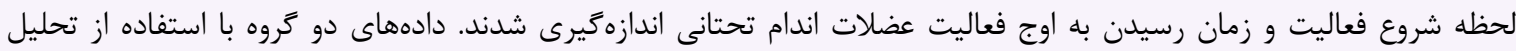

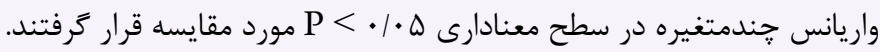

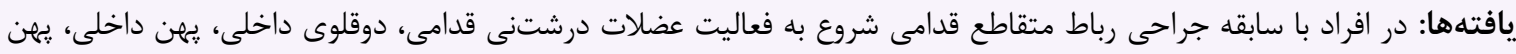

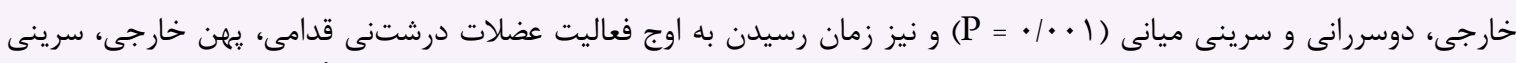

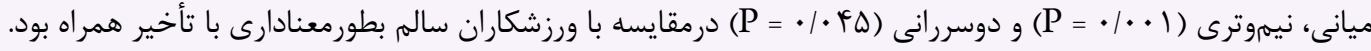

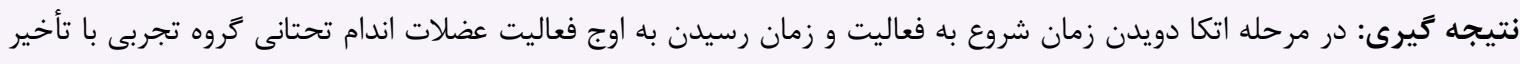

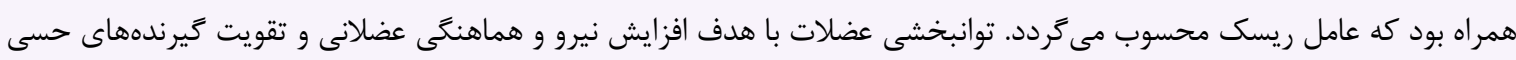

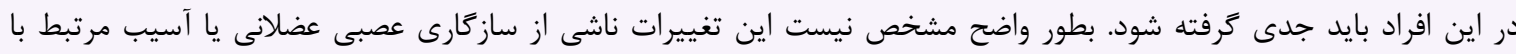

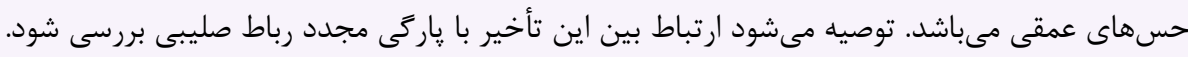

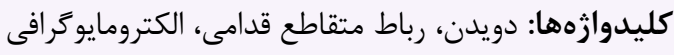

در ورزشكاران جوان صا تا ها ساله شيوع بيشترى دارد [ץ]. نرخ آسيب

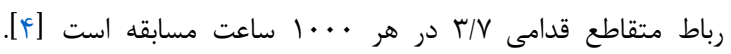

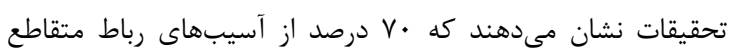

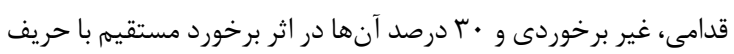

Anterior Cruciate (ACL) هاركى رباط متقاطع قدامى زانو

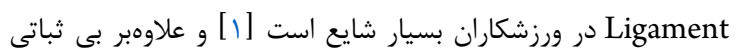

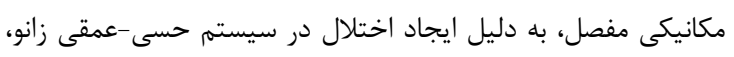

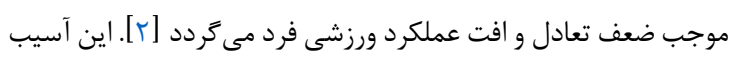


دوقلو يافت نشد؛ همجنين در خانمها با سابقه بازسازى رباط متقاطع

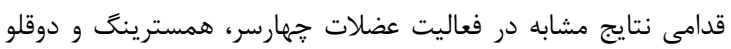

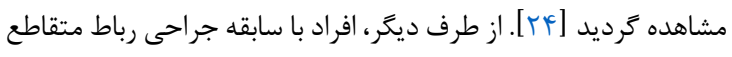

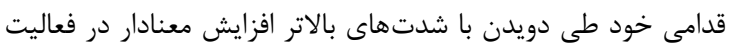

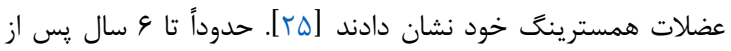

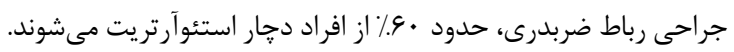

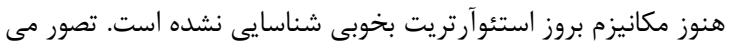

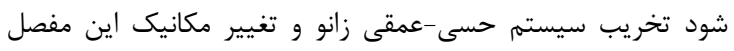

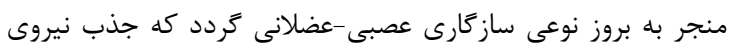

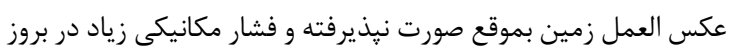

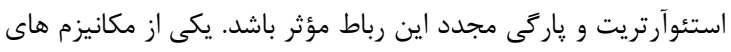

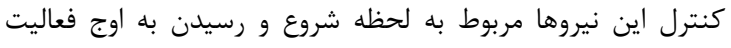

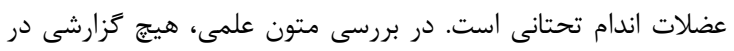

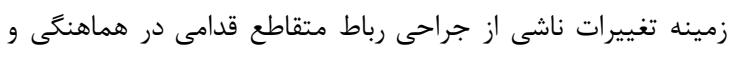

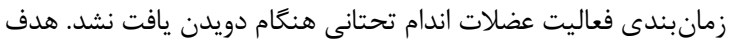

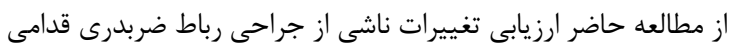

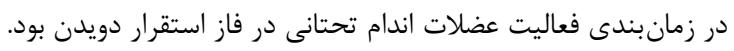

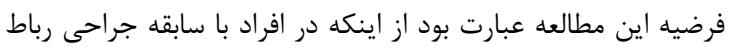

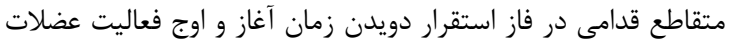
اندام تحتانى با تأخير همراه است.

\section{روش كار}

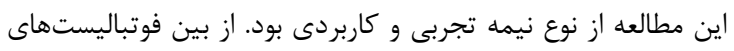

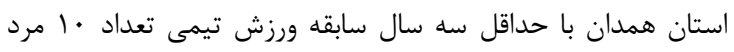
(سن: I/FY

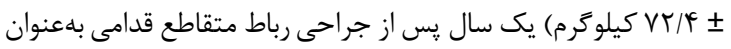

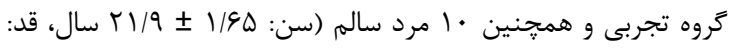

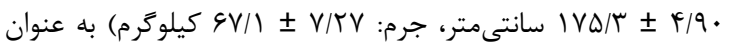

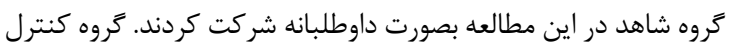

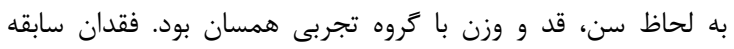
شكستخى استخوان اندام تحتانى و نداشتن ناهنجارى اسكلتى - عضلانى

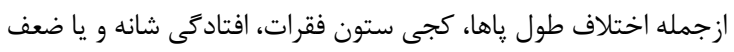

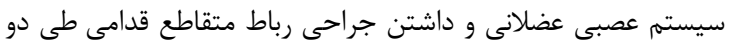

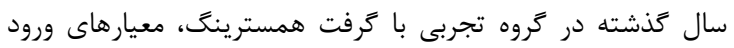

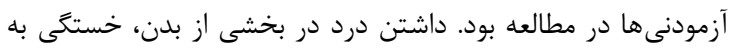

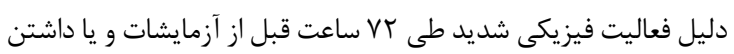

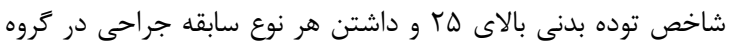

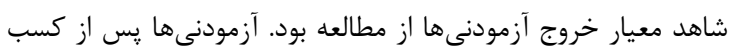

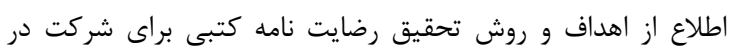

$$
\text { ئزوهش را امضا كردند. }
$$

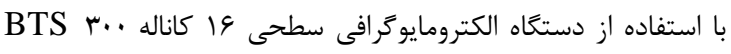
free EMG

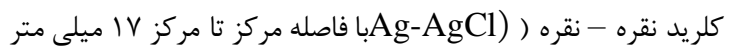

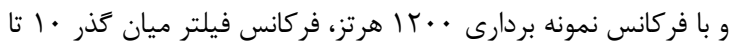

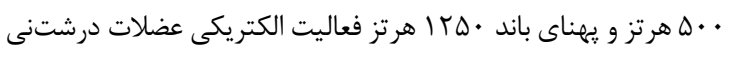

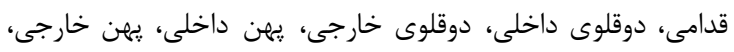

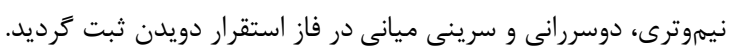

است [ه]. يس از بازسازى رباط متقاطع قدامى ميزان فعاليت و شيوه

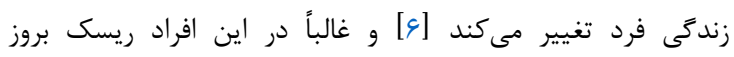

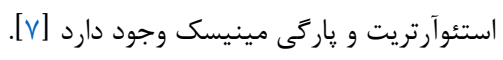

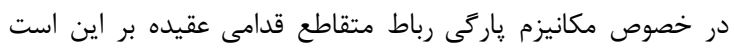

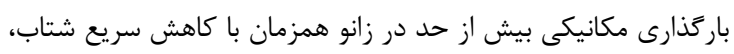

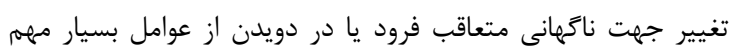

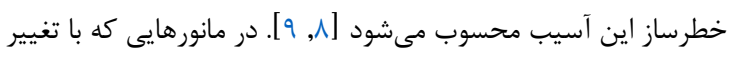

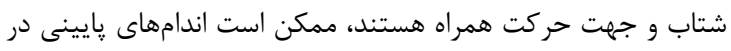

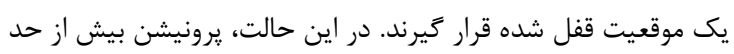

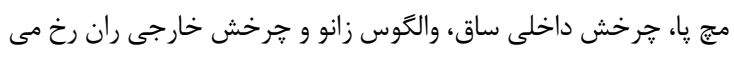

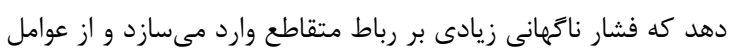

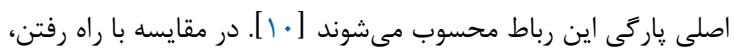

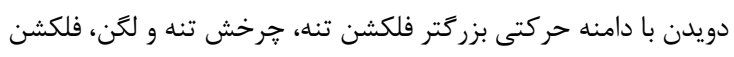

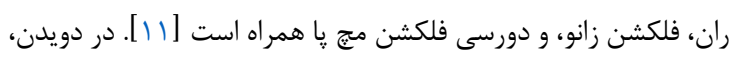

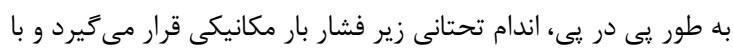

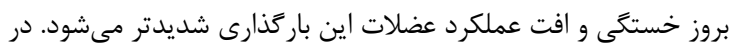

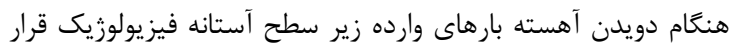

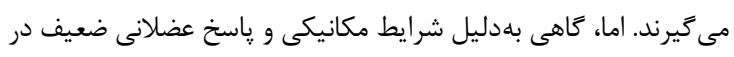

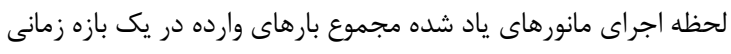

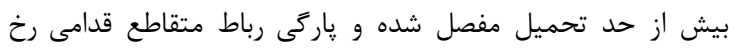

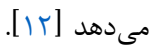
اوج نيروى عكسالعمل عمودى زمين طى فاز استقرار دويدن، در ابتدا

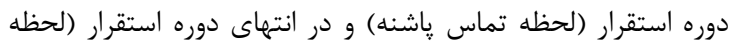

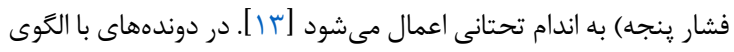

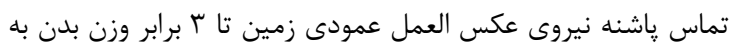

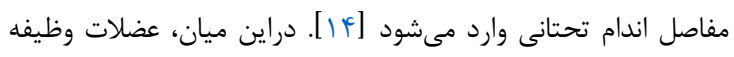

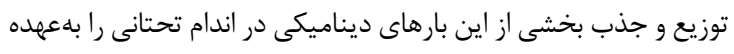

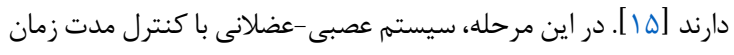

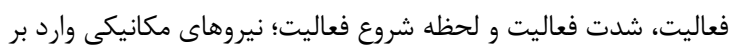

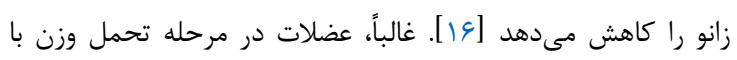

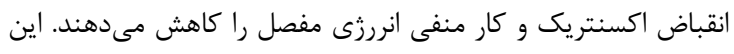

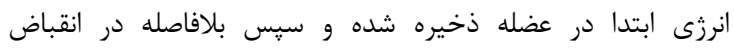

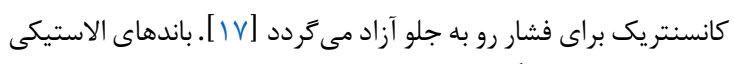

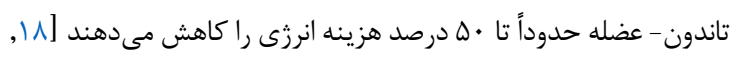

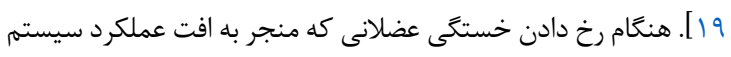

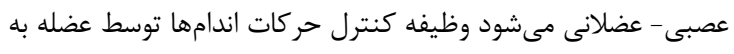

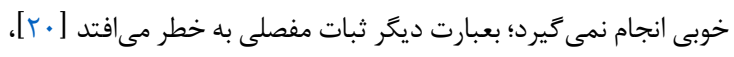

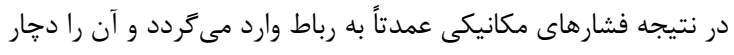

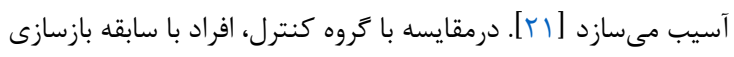

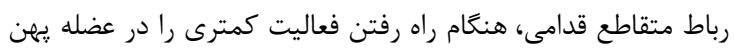

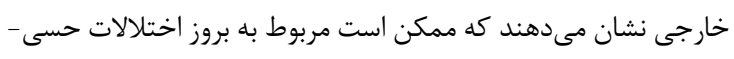

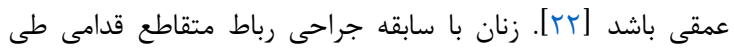

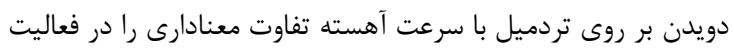

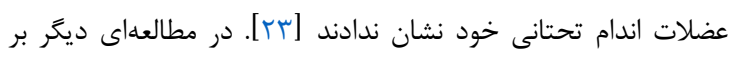

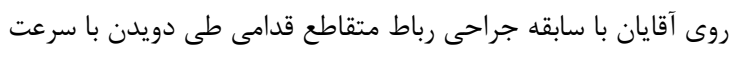

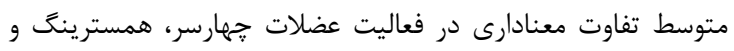


نمودار Linear envelop نقطهى •ا درصدى نسبت به اوج سيكنال

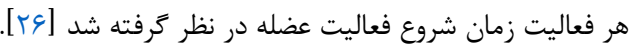

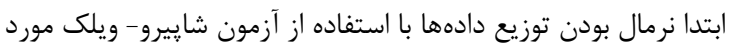

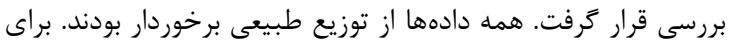

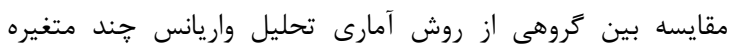
MANOVA

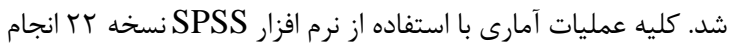

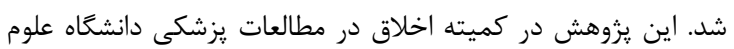

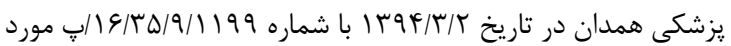

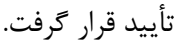

\section{يافتهها}

نتايج تحقيق حاضر نشان داد، زمان شروع به فعاليت عضلات درشتنى درانى دراني

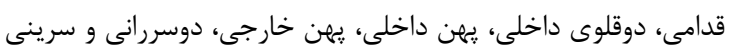

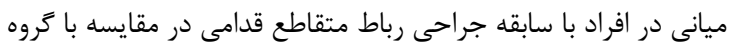

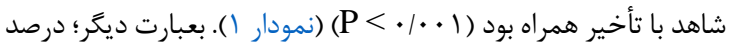

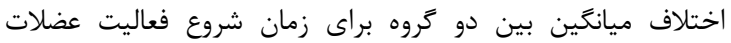

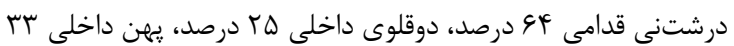

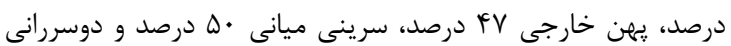

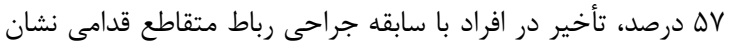

قبل از نصب الكترودها، موهاى روى يوست در محل نصب الكترودها

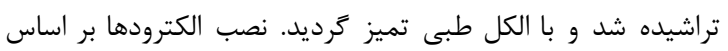

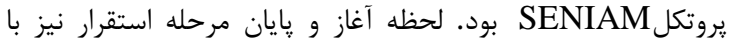

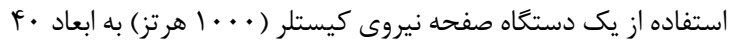

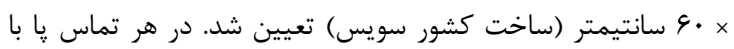

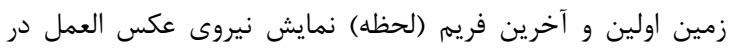

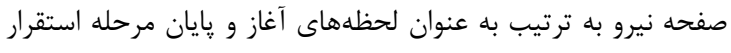

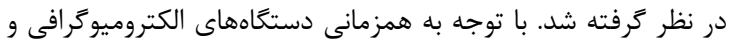

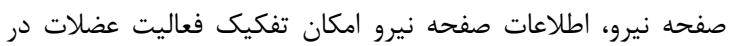
مرحله استقرار را فراهم ميىساخت.

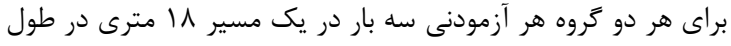

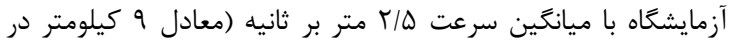

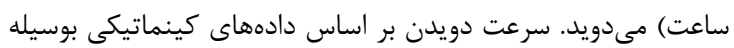

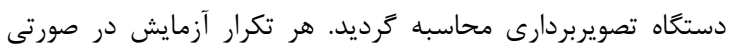

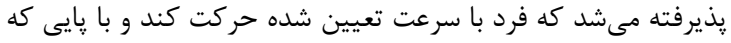

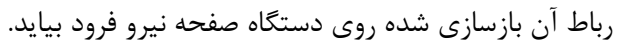

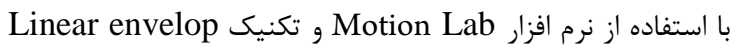

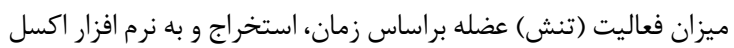

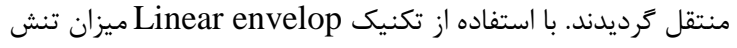

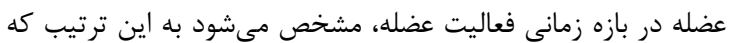

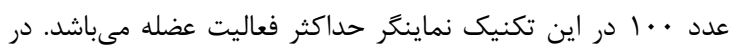

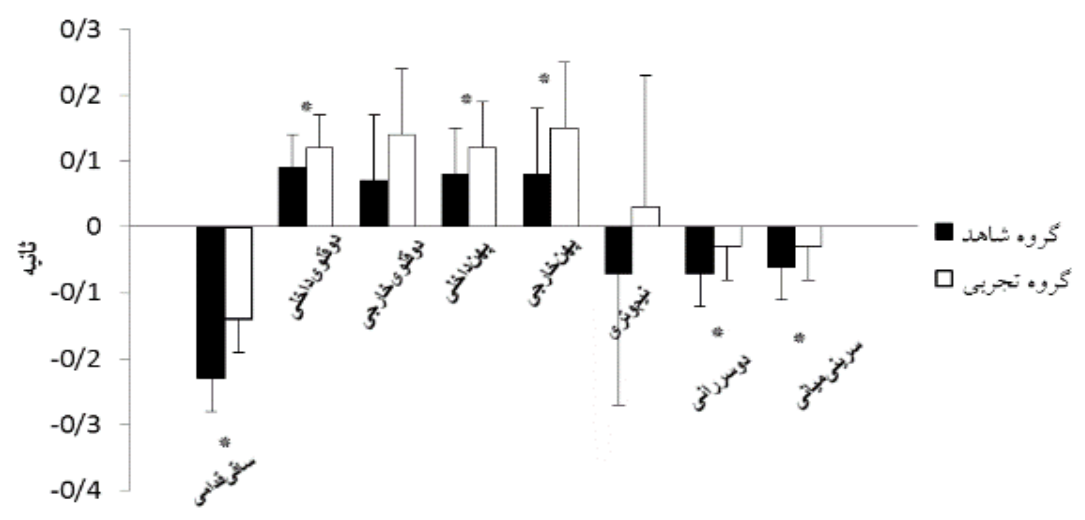

نمودار ا. زمان شروع به فعاليت عضلات در دو كروه شاهد و تجربى (Mean \pm (MD).

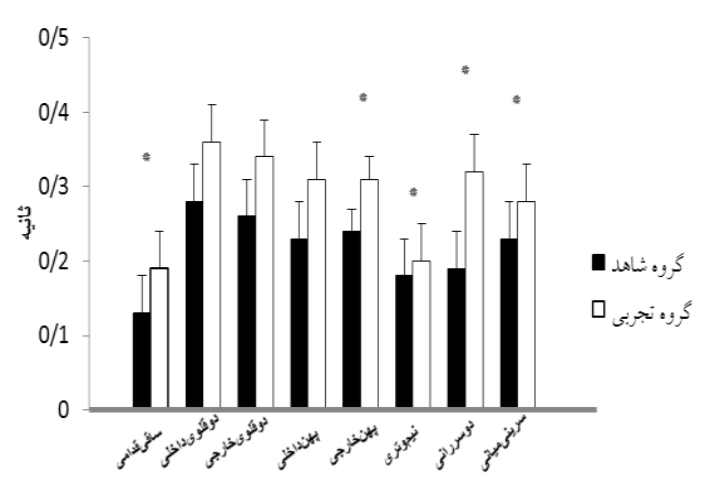

نمودار r. زمان اوج فعاليت عضلات بين در گروه شاهد و تجربى (Mean I SD).
همجنين زمان رسيدن به اوج فعاليت عضلات درشتنىقدامى،

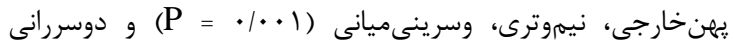

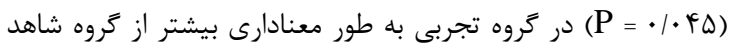

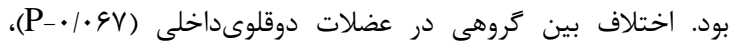

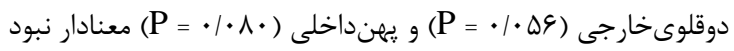

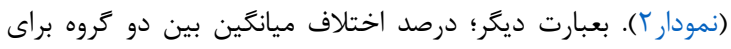

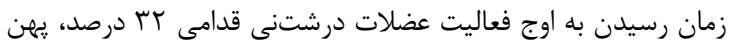

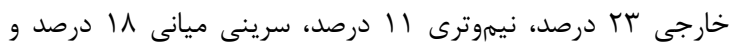

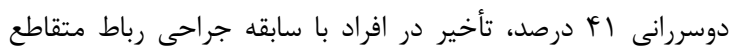

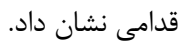


است در جلوَيرى از والكَوس زانو مؤثر باشد و بنابر اين در بيشخَيرى از

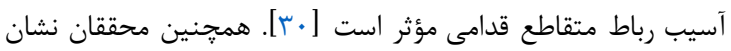

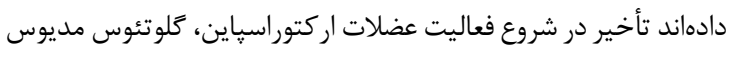

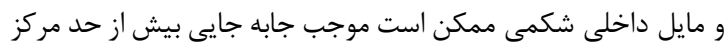

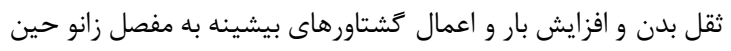

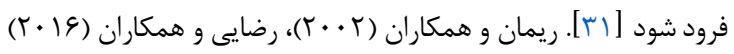

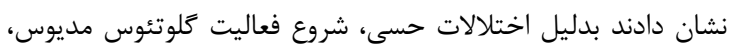

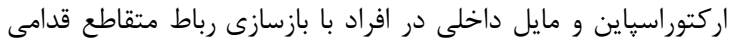

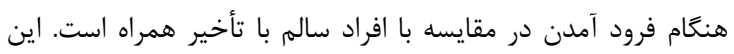

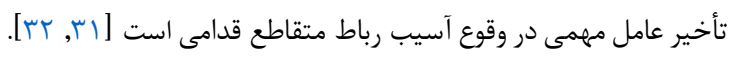

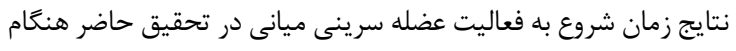

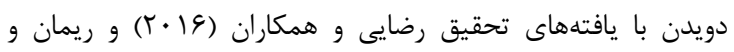

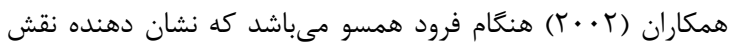

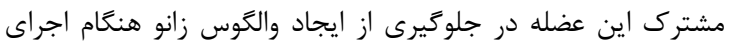
مانورهاى ورزشى با ريسك بالا مى باشد.

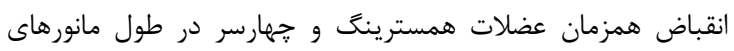

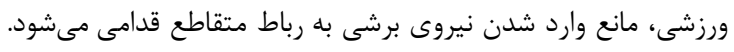

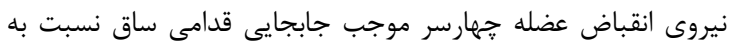

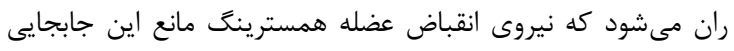

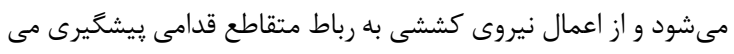

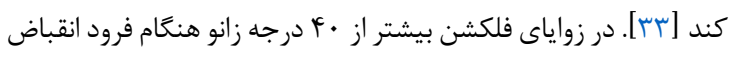

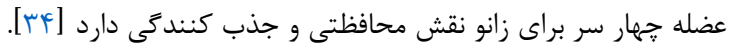

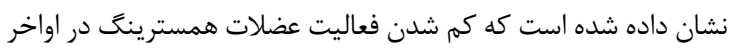

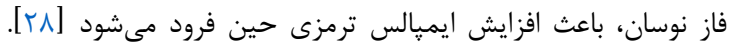

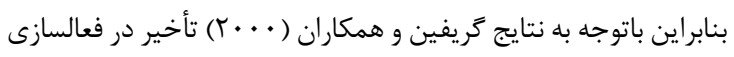

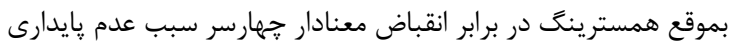

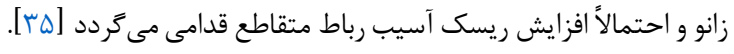

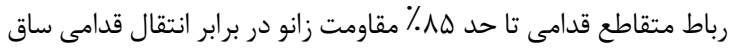

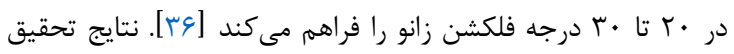

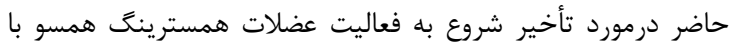

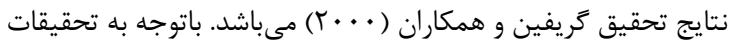

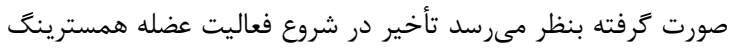

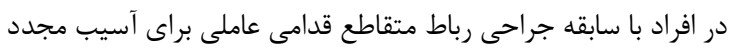

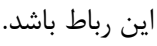

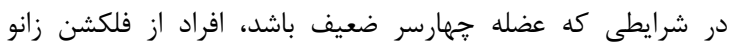

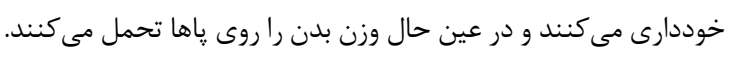

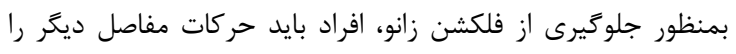

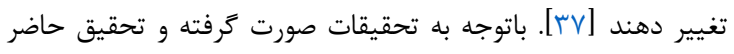

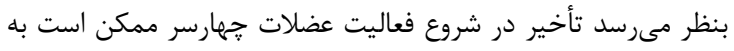

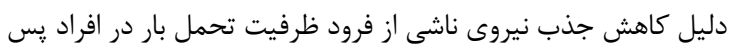

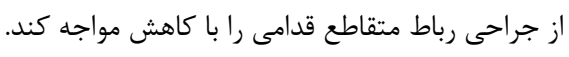

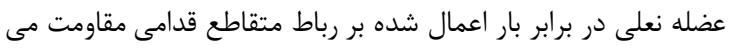

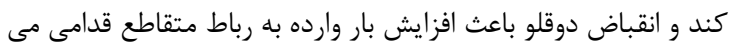

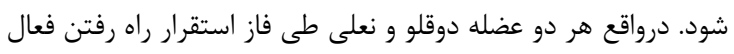

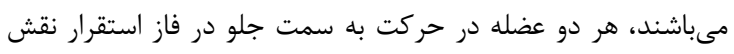

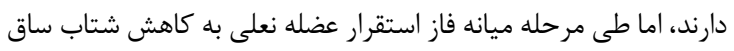

هدف از مطالعه حاضر مقايسه زمانبندى فعاليت عضلات اندام تحتانى

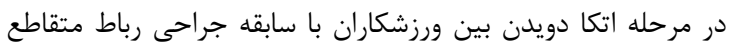

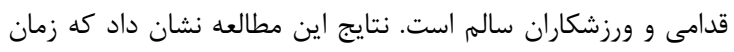

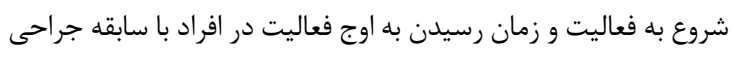

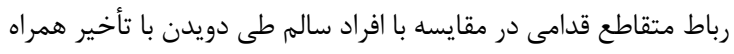

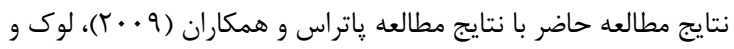

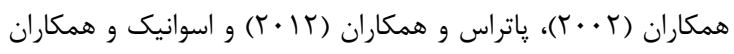

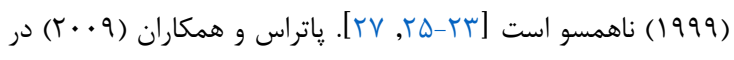

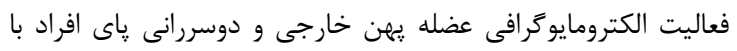

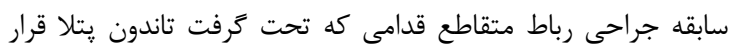

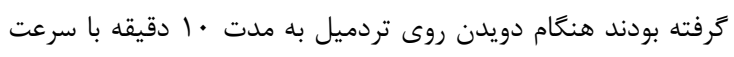

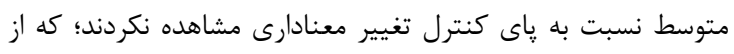

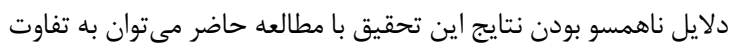

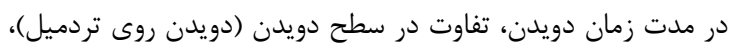

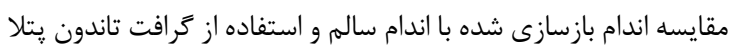

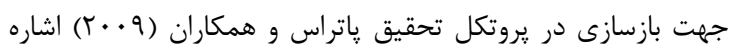

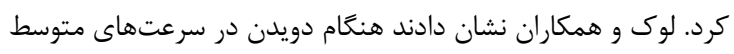

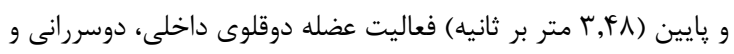

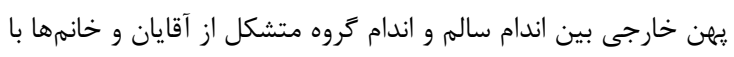

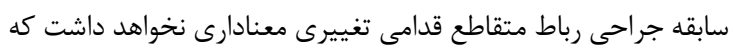

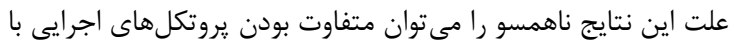

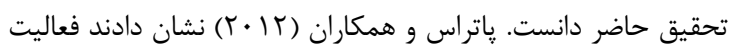

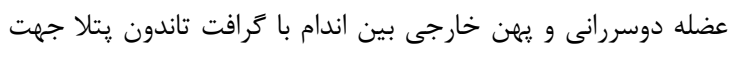

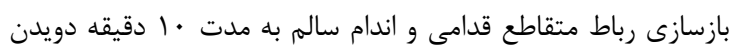

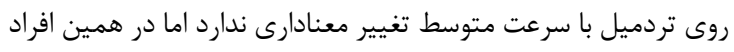

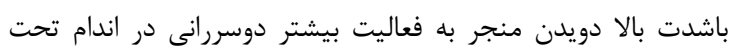

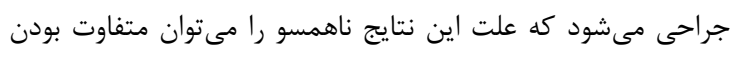

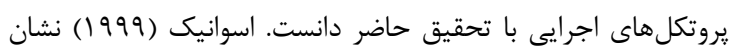

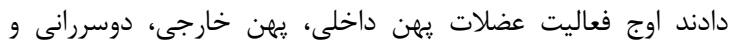

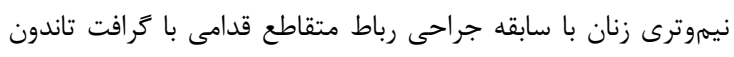

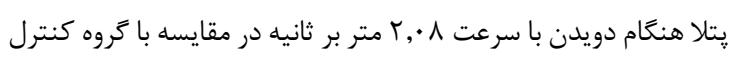

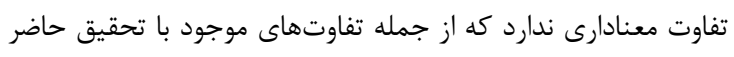

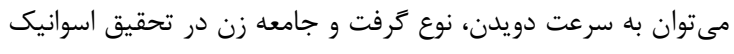

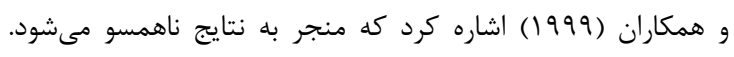

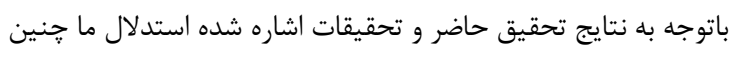

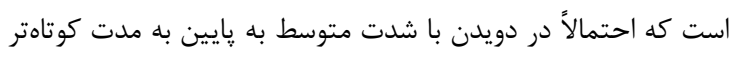

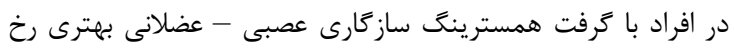

نمى دهد. فعاليت عضلات در اواخر فاز نوسان نقش مهمدى در آماده سازى براى

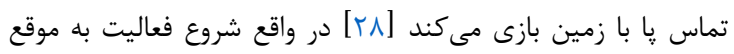

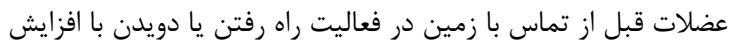

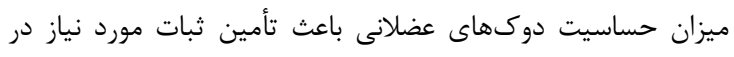

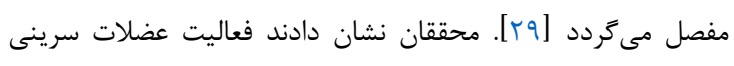
جرخش داخلى ران را در حر كات تحمل وزن كاهش مى دهد كه ممكن فئن 
رباط متقاطع قدامى، اين رباط اطلاعات حسى مهمى را براى درى

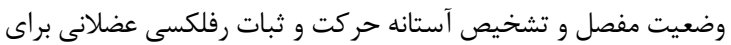

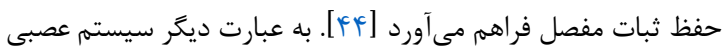

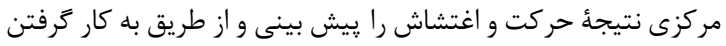

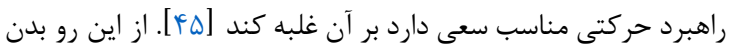

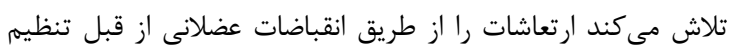

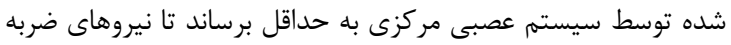

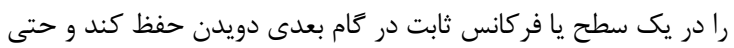

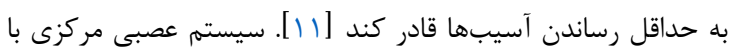

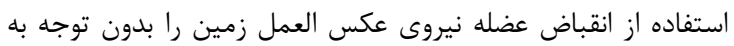

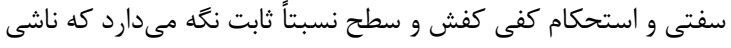

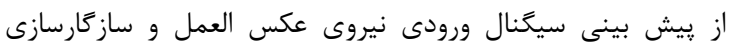

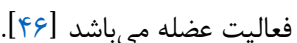

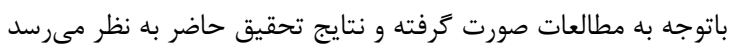

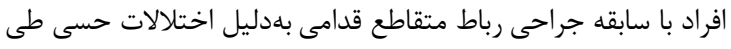

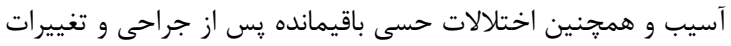

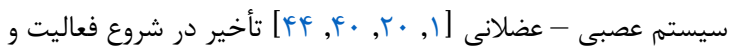

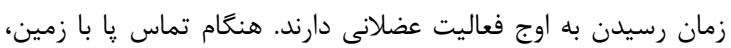

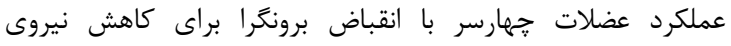

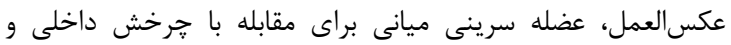

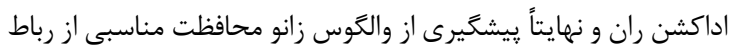

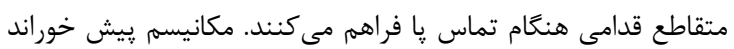

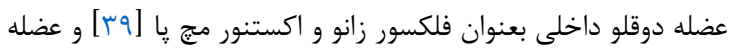

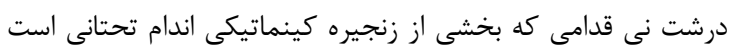

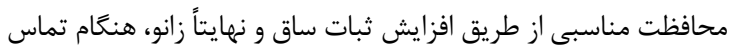

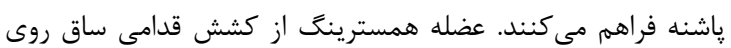

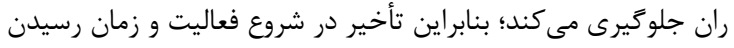

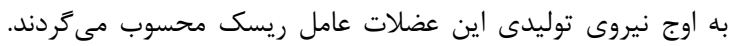

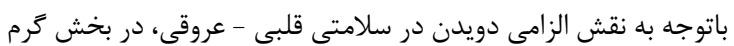

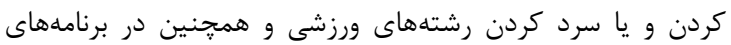

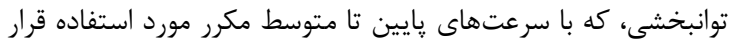

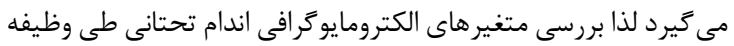

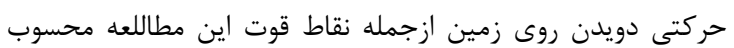

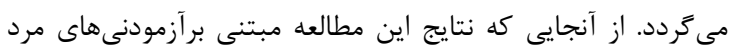

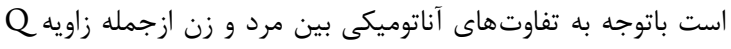

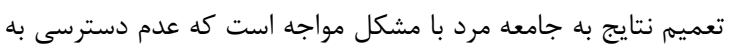

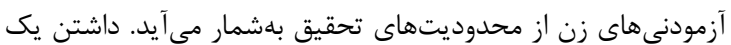

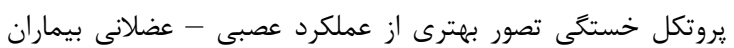

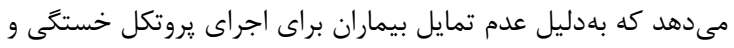

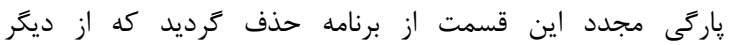
محدوديتهاى تحقيق حاضر محسوب مى كردد.

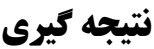

زمان شروع به فعاليت و زمان رسيدن به اوج فعاليت در افراد با سابقه

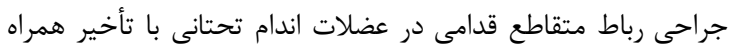

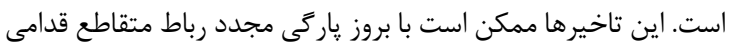

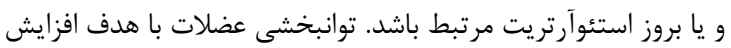

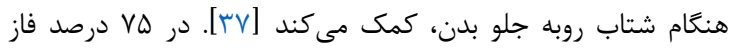

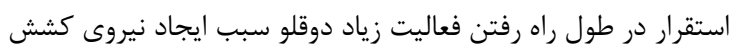

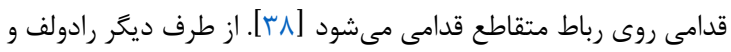

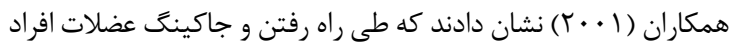

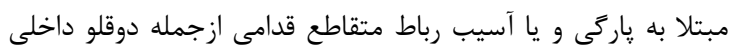

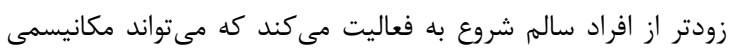

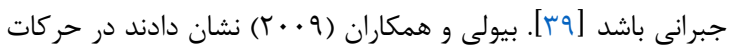

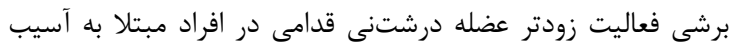

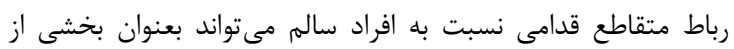

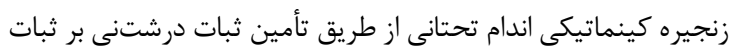

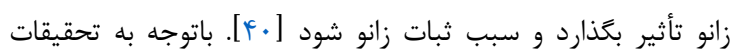

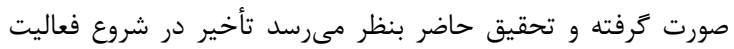

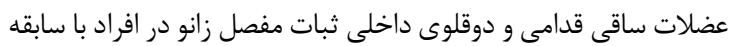

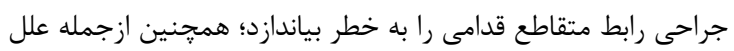

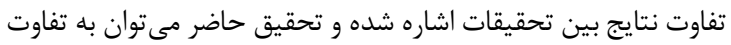
در وظيفه حركتى و جامعه آمارى اشاره كرد.

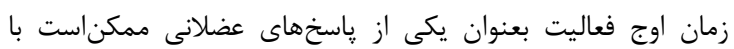

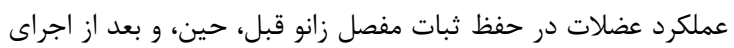

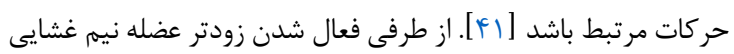

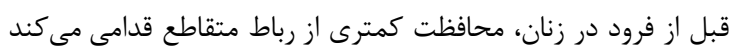

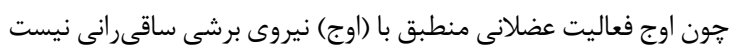

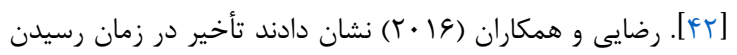

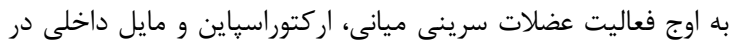

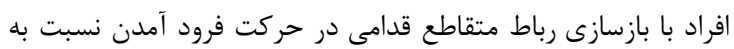

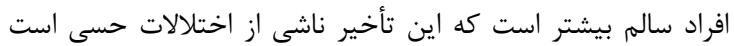

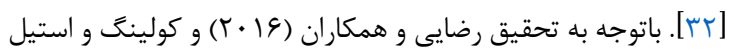

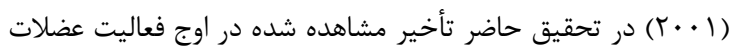

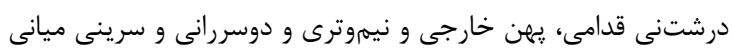

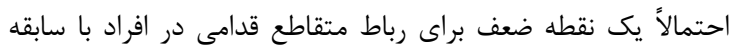

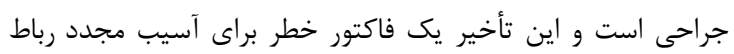

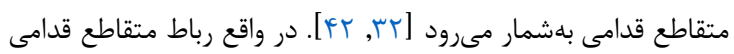

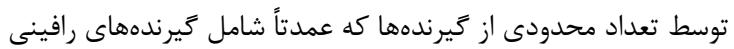

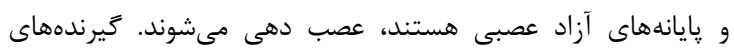

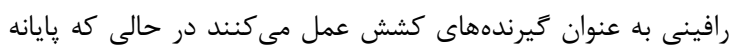

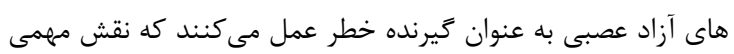

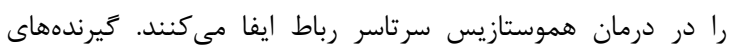

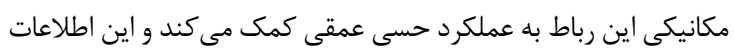

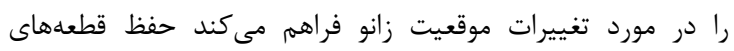

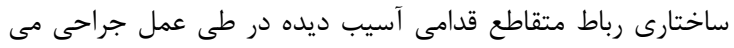

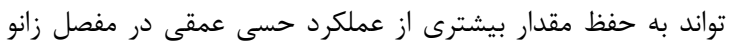

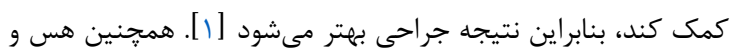

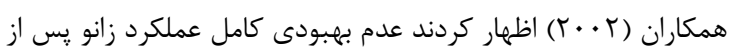

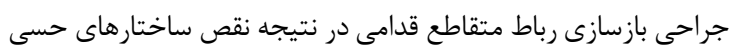

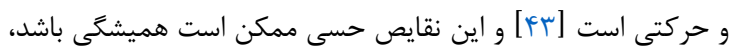

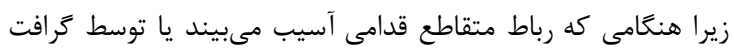

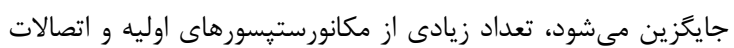

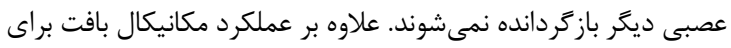




$$
\begin{aligned}
& \text { داد. آقاى ياسين حسينى: ايده اوليه مطالعه و كارهاى آزمايشكاهى و } \\
& \text { رويزن مقاله را انجام داد. } \\
& \text { تضاد منافع } \\
& \text { نويسندًان مقاله هيج گَونه تعارض منافعى را اعلام ندارند. } \\
& \text { كاربرد عملى مطالعه }
\end{aligned}
$$

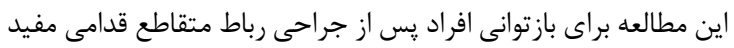

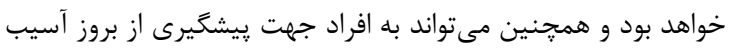

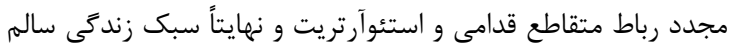

$$
\begin{aligned}
& \text { كمك كند. } \\
& \text { سياسگذارى } \\
& \text { اين مقاله منتج از پايان نامه مقطع كارشناسى ارشد آقاى امين مردآزاد }
\end{aligned}
$$

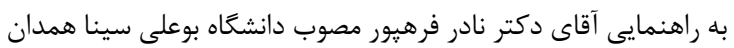

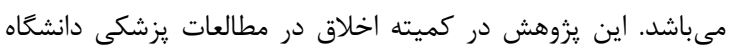

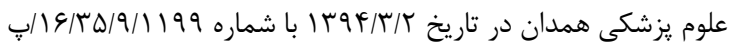

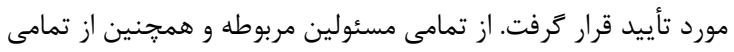

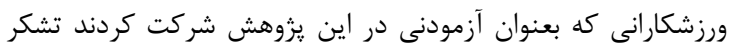

$$
\begin{aligned}
& \text { مى كنيم. }
\end{aligned}
$$

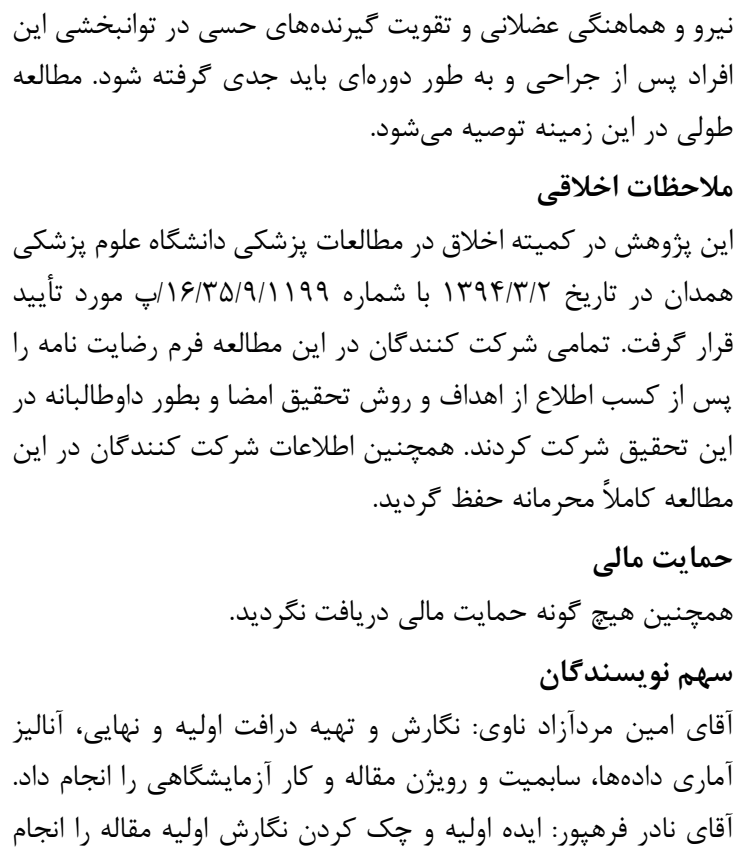

\section{References}

1. Zantop T, Petersen W, Sekiya JK, Musahl V, Fu FH. Anterior cruciate ligament anatomy and function relating to anatomical reconstruction. Knee Surg Sports Traumatol Arthrosc. 2006;14(10):982-92. doi: 10.1007/s00167-006-0076-z pmid: 16897068

2. Gokeler A, Benjaminse A, Hewett TE, Lephart SM, Engebretsen L, Ageberg E, et al. Proprioceptive deficits after ACL injury: are they clinically relevant? Br J Sports Med. 2012;46(3):180-92. doi: 10.1136/bjsm.2010.082578 pmid: 21511738

3. Griffin LY, Albohm MJ, Arendt EA, Bahr R, Beynnon BD, Demaio $M$, et al. Understanding and preventing noncontact anterior cruciate ligament injuries: a review of the Hunt Valley II meeting, January 2005. Am J Sports Med. 2006;34(9):1512-32. doi: 10.1177/0363546506286866 pmid: 16905673

4. Hootman JM, Dick R, Agel J. Epidemiology of collegiate injuries for 15 sports: summary and recommendations for injury prevention initiatives. J Athl Train. 2007;42(2):311-9. pmid: 17710181

5. McNair PJ, Marshall RN, Matheson JA. Important features associated with acute anterior cruciate ligament injury. New Zealand Med J. 1990;103(901):537-9.

6. Grontvedt T, Heir S, Rossvoll I, Engebretsen L. Five-year outcome of 13 patients with an initially undiagnosed anterior cruciate ligament rupture. Scand J Med Sci Sports. 1999;9(1):62-4. doi: 10.1111/j.16000838.1999.tb00209.x pmid: 9974200

7. Sell TC, Ferris CM, Abt JP, Tsai YS, Myers JB, Fu FH, et al. Predictors of proximal tibia anterior shear force during a vertical stop-jump. J Orthop Res. 2007;25(12):1589-97. doi: 10.1002/jor.20459 pmid: 17626264

8. Boden BP, Dean GS, Feagin JA, Jr., Garrett WE, Jr. Mechanisms of anterior cruciate ligament injury. Orthopedics. 2000;23(6):573-8. pmid: 10875418
9. McLean SG, Huang X, van den Bogert AJ. Investigating isolated neuromuscular control contributions to noncontact anterior cruciate ligament injury risk via computer simulation methods. Clin Biomech (Bristol, Avon). 2008;23(7):926-36.

doi:

10.1016/j.clinbiomech.2008.03.072 pmid: 18485552

10. Ireland ML. Anterior cruciate ligament injury in female athletes: epidemiology. J Athl Train. 1999;34(2):150-4. pmid: 16558558

11. Lohman EB, 3rd, Balan Sackiriyas KS, Swen RW. A comparison of the spatiotemporal parameters, kinematics, and biomechanics between shod, unshod, and minimally supported running as compared to walking. Phys Ther Sport. 2011;12(4):151-63. doi: 10.1016/j.ptsp.2011.09.004 pmid: 22085708

12. Hohmann E, Wortler $K$, Imhoff $A B$. MR imaging of the hip and knee before and after marathon running. Am J Sports Med. 2004;32(1):55-9. doi: 10.1177/0363546503258904 pmid: 14754724

13. Cavanagh PR, Lafortune MA. Ground reaction forces in distance running. J Biomechanic. 1980;13(5):397-406. doi: 10.1016/0021-9290(80)90033-0

14. Lieberman DE, Venkadesan M, Werbel WA, Daoud AI, D'Andrea S, Davis IS, et al. Foot strike patterns and collision forces in habitually barefoot versus shod runners. Nature. 2010;463(7280):531-5. doi: 10.1038/nature08723 pmid: 20111000

15. Radin EL. Role of muscles in protecting athletes from injury. Acta Med Scand Suppl. 1986;711:143-7. doi: 10.1111/j.0954-6820.1986.tb08943.x pmid: 3465203

16. Komi PV. Stretch-shortening cycle: a powerful model to study normal and fatigued muscle. J Biomechanic. 2000;33(10):1197-206. doi: 10.1016/S00219290(00)00064-6 
17. Cavanagh PR, Kram R. Stride length in distance running: velocity, body dimensions, and added mass effects. Med Sci Sports Exerc. 1989;21(4):467-79. doi: 10.1249/00005768-198908000-00020

18. Alexander RM. Energy-saving mechanisms in walking and running. J Experimental Biol. 1991;160(1):55-69.

19. Alexander RM. Models and the scaling of energy costs for locomotion. J Exp Biol. 2005;208(Pt 9):1645-52. doi: 10.1242/jeb.01484 pmid: 15855396

20. De Ste Croix MB, Priestley AM, Lloyd RS, Oliver JL. ACL injury risk in elite female youth soccer: Changes in neuromuscular control of the knee following soccerspecific fatigue. Scand J Med Sci Sports. 2015;25(5):e531-8. doi: 10.1111/sms.12355 pmid: 25556396

21. Jacobs CA, Uhl TL, Mattacola CG, Shapiro R, Rayens WS. Hip abductor function and lower extremity landing kinematics: sex differences. J Athl Train. 2007;42(1):7683. pmid: 17597947

22. Leporace G, Metsavaht L, Pereira GR, Oliveira LP, Crespo B, Batista LA. Knee Synergism during Gait Remain Altered One Year after Acl Reconstruction. Acta Ortop Bras. 2016;24(3):137-41. doi: 10.1590/1413785220162403153479 pmid: 27217814

23. Swanik CB, Lephart SM, Giraldo JL, DeMont RG, Fu FH. Reactive muscle fring of anterior cruciate ligamentinjured females during functional activities. J Athl Train. 1999;34:121-9. doi: 10.1007/s40279-019-01120-x pmid: 31102111

24. Lewek M, Rudolph K, Axe M, Snyder-Mackler L. The efect of insufcient quadriceps strength on gait after anterior cruciate ligament reconstruction. Clin Biomech. 2002;17:56-63. doi: 10.1007/s40279-019-01120-x pmid: 31102111

25. Patras K, Zampeli F, Ristanis S, Tsepis E, Ziogas G, Stergiou N. Hamstring-dominant strategy of the bonepatellar tendon-bone graft anterior cruciate ligamentreconstructed leg versus quadriceps-dominant strategy of the contralateral intact leg during high-intensity exercise in male athletes. Arthroscopy. 2012;28:1262-70. doi: 10.1007/s40279-019-01120-x pmid: 31102111

26. O'Connor KM, Price TB, Hamill J. Examination of extrinsic foot muscles during running using $\mathrm{mfMRI}$ and EMG. J Electromyogr Kinesiol. 2006; 16(5):522-30. doi: 10.1016/j.jelekin.2005.09.003 pmid: 16275015

27. Patras K, Ziogas G, Ristanis S, Tsepis E, Stergiou N, Georgoulis $\mathrm{AD}$. High intensity running results in an impaired neuromuscular response in ACL reconstructed individuals. Knee Surg Sports Traumatol Arthrosc. 2009;17:977-84. doi: 10.1007/s40279-019-01120-x pmid: 31102111

28. Kyrolainen H, Belli A, Komi PV. Biomechanical factors affecting running economy. Med Sci Sports Exerc. 2001;33(8):1330-7. doi: 10.1097/00005768200108000-00014 pmid: 11474335

29. Brown TN, Palmieri-Smith RM, McLean SG. Sex and limb differences in hip and knee kinematics and kinetics during anticipated and unanticipated jump landings: implications for anterior cruciate ligament injury. $\mathrm{Br} \mathrm{J}$ Sports Med. 2009;43(13):1049-56. doi: 10.1136/bjsm.2008.055954 pmid: 19372596

30. Kagaya Y, Fujii Y, Nishizono H. Association between hip abductor function, rear-foot dynamic alignment, and dynamic knee valgus during single-leg squats and drop landings. J Sport Health Sci. 2015;4(2):182-7. doi: 10.1016/j.jshs.2013.08.002

31. Riemann BL, Lephart SM. The sensorimotor system, part I: the physiologic basis of functional joint stability. J Athl Train. 2002;37(1):71-9. pmid: 16558670

32. Rezaiee K, Mazloum V, Mamashli A. A comparison of timing of core muscles between ACL reconstructed and healthy athletes while landing. (Persian). J Sport Med. 2016;8(1):49-65.

33. DeMorat G, Weinhold P, Blackburn T, Chudik S, Garrett $\mathrm{W}$. Aggressive quadriceps loading can induce noncontact anterior cruciate ligament injury. Am J Sports Med. 2004;32(2):477-83. doi: 10.1177/0363546503258928 pmid: 14977677

34. Chappell JD, Yu B, Kirkendall DT, Garrett WE. A comparison of knee kinetics between male and female recreational athletes in stop-jump tasks. Am J Sports Med. 2002;30(2):261-7. doi: 10.1177/03635465020300021901 pmid: 11912098

35. Griffin LY, Agel J, Albohm MJ, Arendt EA, Dick RW, Garrett WE, et al. Noncontact anterior cruciate ligament injuries: risk factors and prevention strategies. J Am Acad Orthop Surg. 2000;8(3):141-50. doi: 10.5435/00124635-200005000-00001 pmid: 10874221

36. Markolf KL, Burchfield DM, Shapiro MM, Shepard MF, Finerman GA, Slauterbeck JL. Combined knee loading states that generate high anterior cruciate ligament forces. J Orthop Res. 1995;13(6):930-5. doi: 10.1002/jor.1100130618 pmid: 8544031

37. Oatis CA. Kinesiology: the mechanics and pathomechanics of human movement. Philadelphia: Lippincott Williams \& Wilkins; 2004.

38. Adouni M, Shirazi-Adl A, Marouane H. Role of gastrocnemius activation in knee joint biomechanics: gastrocnemius acts as an ACL antagonist. Comput Methods Biomech Biomed Engin. 2016;19(4):376-85. doi: 10.1080/10255842.2015.1032943 pmid: 25892616

39. Rudolph KS, Axe MJ, Buchanan TS, Scholz JP, SnyderMackler L. Dynamic stability in the anterior cruciate ligament deficient knee. Knee Surg Sports Traumatol Arthrosc. 2001;9(2):62-71. doi: $10.1007 /$ s001670000166 pmid: 11354855

40. Hess T, Duchow J, Roland S, Kohn D. Single-versus twoincision technique in anterior cruciate ligament replacement: influence on postoperative muscle function. Am J Sports Med. 2002;30(1):27-31. doi: 10.1177/03635465020300011801 pmid: 11798992

41. Coats-Thomas MS, Miranda DL, Badger GJ, Fleming BC. Effects of ACL reconstruction surgery on muscle activity of the lower limb during a jump-cut maneuver in males and females. J Orthop Res. 2013;31(12):1890-6. doi: 10.1002/jor.22470 pmid: 23966333 
42. Cowling EJ, Steele JR. Is lower limb muscle synchrony during landing affected by gender? Implications for variations in ACL injury rates. J Electromyograph Kinesiol. 2001;11(4):263-8. doi: 10.1016/S10506411(00)00056-0

43. Beaulieu ML, Lamontagne M, Xu L. Lower limb muscle activity and kinematics of an unanticipated cutting manoeuvre: a gender comparison. Knee Surg Sports Traumatol Arthrosc. 2009;17(8):968-76. doi: 10.1007/s00167-009-0821-1 pmid: 19495727

44. Lee HM, Cheng CK, Liau JJ. Correlation between proprioception, muscle strength, knee laxity, and dynamic standing balance in patients with chronic anterior cruciate ligament deficiency. Knee. 2009;16(5):387-91. doi: 10.1016/j.knee.2009.01.006 pmid: 19239988

45. Huijbregts PA. Therapeutic exercise for lumbopelvic stabilization: A motor control approach for the treatment and prevention of low back pain, ed 2. Physic Therap. 2005;85(5):470.

46. Zadpoor AA, Nikooyan AA. Modeling muscle activity to study the effects of footwear on the impact forces and vibrations of the human body during running. J Biomech. 2010;43(2):186-93.

doi: 